\title{
Polarization anisotropy of charge transfer absorption and emission of aligned polymer: fullerene blend films
}

Koen Vandewal, Kristofer Tvingstedt and Olle Inganäs

\section{Linköping University Post Print}

N.B.: When citing this work, cite the original article.

Original Publication:

Koen Vandewal, Kristofer Tvingstedt and Olle Inganäs, Polarization anisotropy of charge transfer absorption and emission of aligned polymer: fullerene blend films, 2012, Physical Review B. Condensed Matter and Materials Physics, (86), 3, 035212.

http://dx.doi.org/10.1103/PhysRevB.86.035212

Copyright: American Physical Society http://www.aps.org/

Postprint available at: Linköping University Electronic Press

http://urn.kb.se/resolve?urn=urn:nbn:se:liu:diva-81833 


\title{
Polarization anisotropy of charge transfer absorption and emission of aligned polymer: fullerene blend films
}

\author{
Koen Vandewal, ${ }^{*}$ Kristofer Tvingstedt, and Olle Inganäs \\ Biomolecular and Organic Electronics, IFM, Linköping University, SE-581 83 Linköping, Sweden
}

(Received 25 May 2012; published 30 July 2012)

\begin{abstract}
An improved understanding of the electronic structure of interfacial charge transfer (CT) states is of importance due to their crucial role in charge carrier generation and recombination in organic donor-acceptor $(D A)$ solar cells. $D A$ combinations with a small difference between the energy of the CT state $\left(E_{\mathrm{CT}}\right)$ and energy of the donor exciton $\left(E_{D^{*}}\right)$ are of special interest since energy losses due to electron transfer are minimized, resulting in an optimized open-circuit voltage. In that case, the CT state can be considered as a resonance mixture, containing character of a fully ionic state $\left(D^{+} A^{-}\right)$and of the local polymer excited state $\left(D^{*} A\right)$. We show that the $D^{*} A$ contribution to the overall $\mathrm{CT}$ state wave function can be determined by measurements of the polarization anisotropy of CT absorption and emission of polymer:fullerene blends with aligned polymer chains. We study two donor polymers, P3HT and TQ1, blended with fullerene acceptors with different ionization potentials, allowing variation of the $E_{D^{*}}-E_{\mathrm{CT}}$ difference. We find that, upon decreasing $E_{D^{*}}-E_{\mathrm{CT}}$, the local excitonic $D^{*} A$ character of the CT state increases, resulting in a decreased fraction of charge transferred and an increased transition dipole moment. For typical polymer:fullerene systems, this effect is expected to become detrimental for device performance if $E_{D^{*}}-E_{\mathrm{CT}}<0.1 \mathrm{eV}$. This however, depends on the electronic coupling between $D^{*} A$ and $D^{+} A^{-}$, which we experimentally estimate to be $\sim 6 \mathrm{meV}$ for the TQ1:PCBM system.
\end{abstract}

DOI: 10.1103/PhysRevB.86.035212

PACS number(s): 73.50.Pz, 78.66.Qn, 88.40.jr

\section{INTRODUCTION}

A detailed understanding of the mechanisms of charge generation and recombination at electron donor-acceptor $(D A)$ organic-organic interfaces will benefit the development of organic optoelectronic applications, such as light-emitting diodes and solar cells. Some polymer-polymer interfaces are quite efficient light emitters, with the emitted light originating from the interfacial excited state, often termed exciplex emission. ${ }^{1-4}$ On the other hand, the polymer-fullerene interface, exploited extensively in organic solar cells, can exhibit efficient charge carrier generation, ${ }^{5-7}$ but is, generally, a poor light emitter. ${ }^{8-11}$ Theoretical and experimental determination of the electronic structure of such organic-organic interfaces can elucidate on the coupling between the relevant states, rate constants, and efficiencies of charge carrier generation and recombination. ${ }^{1,12,13}$ Such work is crucial for both the understanding and improvement of devices based on blends of organic materials.

Efficient charge carrier generation at a donor-acceptor interface implies that the forward electron transfer from excited donor $(D)$ to acceptor $(A)$ material is fast as compared to pure donor exciton $\left(D^{*}\right)$ decay. It further implies that the resulting charge transfer (CT) state is unstable and that the initial electron transfer is followed by additional electron transfer steps in order to separate the charge carriers further. In efficient solar cells, these charge-separating electron transfer steps are faster than the back electron transfer step, i.e., decay of the CT state to the ground state $(D A) .{ }^{14}$ Once charge carriers are free, there is a probability that positive and negative charge carriers will meet at an interface, resulting again in the formation of a CT state, which can redissociate or recombine. At open circuit, this type of recombination balances with the free carrier generation, ${ }^{15}$ hence the open-circuit voltage $V_{o c}$ of donor-acceptor solar cells is found to be related to properties of the interfacial CT state. ${ }^{16-18} V_{o c}$ under solar illumination can be linearly correlated with the energy of the CT state $\left(E_{\mathrm{CT}}\right) .{ }^{19-21}$ In order to maximize photovoltaic energy conversion efficiency, polymer:fullerene combinations with a high $E_{\mathrm{CT}}$ and low optical gap of the donor $E_{D^{*}}$ (or main light absorber) are of particular relevance since such properties optimize $V_{o c}$ by minimizing the energetic loss due to the electron transfer from $D$ to $A$ and maximizing the amount of photons harvested. The question as to whether it is possible to maintain efficient free charge carrier generation and collection (internal quantum efficiencies $>90 \%$ ) under the condition of a small $E_{D^{*}}-E_{\mathrm{CT}}$ difference, however, is still open. ${ }^{14,22}$ For example, for such low driving force material systems, triplet energies of polymer and/or fullerene can be below the CT state, sparking debate as to whether population of the triplet state in such systems constitutes a fundamental loss. ${ }^{23-25}$ Another topic of intense debate relates to the excess energy from excited donor states and if it is exploited as kinetic energy to increase the intercharge pair separation distance, improving the free charge carrier generation rate. ${ }^{25-31}$

Therefore, theoretical and experimental investigations of the electronic structure of the CT state, focusing on the influence of the $E_{D^{*}}-E_{\mathrm{CT}}$ difference, are of interest. The electronic structure of excited states is intimately linked to the rates and spectral position of optical absorption and emission. ${ }^{32}$ In this respect, the study of CT absorption and emission has already proven useful for the determination of CT state energies for different polymer:fullerene combinations. ${ }^{21}$ Furthermore, donor-acceptor separation distance ${ }^{33}$ and crystallization of either polymer ${ }^{34}$ or fullerene ${ }^{35}$ phase have a pronounced influence on the spectral position and strength of CT absorption bands.

In this work, we explore the relation between electronic structure of the CT state and CT absorption and emission 
(a)

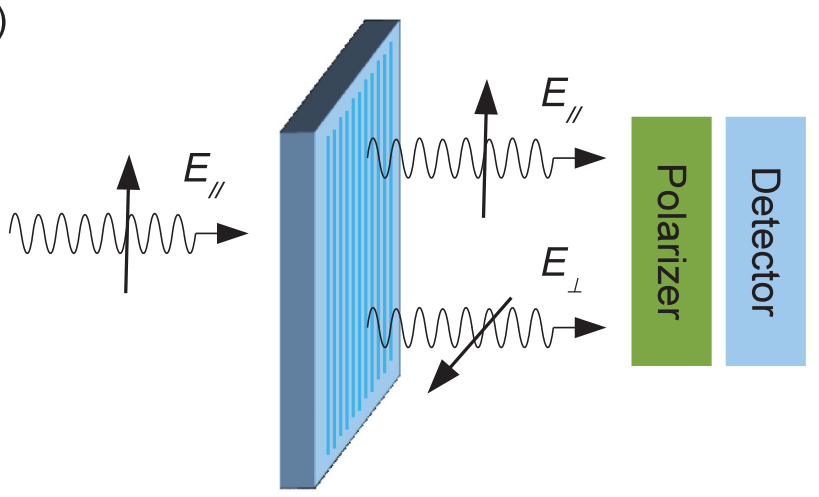

(b)

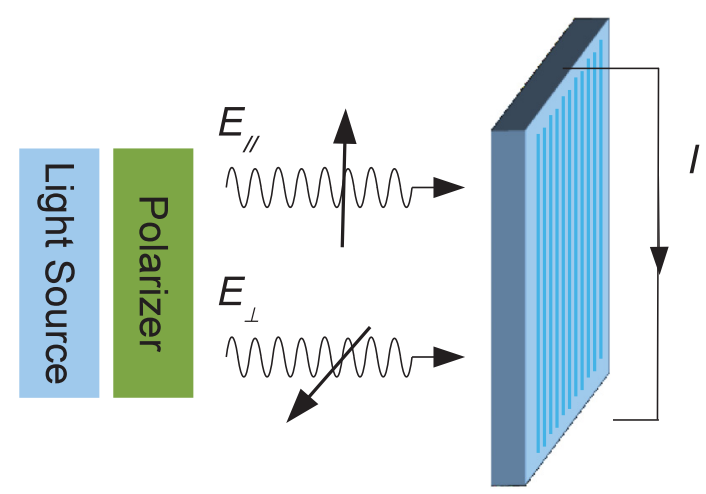

FIG. 1. (Color online) Schematic representation of the experiments. (a) In the PL experiments, the rubbed sample is excited with a laser $(405 \mathrm{~nm})$ with polarization parallel to the direction of polymer alignment. The polarizer in front of the detector allows detection of the emitted spectrum with polarizations parallel and perpendicular to the direction of alignment. (b) For the photocurrent measurements, light passes through a polarizer to set the direction of polarization parallel or perpendicular to the direction of polymer chain alignment. In this work, the photocurrent spectrum is obtained by using the technique Fourier-transform photocurrent spectroscopy (FTPS), in which the light source is provided by the output beam of an Fourier-transform infrared spectrometer (FTIR), equipped with a halogen lamp and quartz beam splitter.

further. We study the polarization anisotropy of CT absorption and emission of polymer:fullerene blend films with the polymer chains aligned by mechanical rubbing (Fig. 1). We use three different fullerene derivatives: PCBM, bisPCBM, ${ }^{36}$ and HG2-V2, ${ }^{37}$ combined with the donor polymers TQ1 (Ref. 38) and P3HT. The different lowest unoccupied molecular orbitals (LUMOs) of the fullerene derivatives allows us to vary $E_{D^{*}}-E_{\mathrm{CT}}$ for blends with each donor polymer. Photoluminescence experiments allow the comparison of the polarization anisotropy of the CT state and the pure donor excitation for films of TQ1:fullerene blends. Photovoltaic devices comprising rubbing aligned active layers of TQ1:fullerene blends and P3HT:fullerene blends allow for the measurement of polarization anisotropy in the photocurrent spectrum. The use of the sensitive technique of Fouriertransform photocurrent spectroscopy (FTPS) enables us to compare the anisotropy in the low-energy spectral region of the weak CT absorption to the anisotropy of the stronger fullerene and polymer absorption.
For all material blends, we find that the transition dipole moment of the $\mathrm{CT}$ transition has a substantial component in the direction of polymer alignment. Moreover, this component increases with decreasing $E_{D^{*}}-E_{\mathrm{CT}}$. The results are interpreted by considering the CT state as a resonance between a pure donor excited state $\left(D^{*} A\right)$ and a fully charge transferred state $\left(D^{+} A^{-}\right) .^{1,39,40}$ In this simple picture, the overall electronic structure of the CT state depends on $E_{D^{*}}-E_{\mathrm{CT}}$ and the coupling matrix element $V^{*}$, mixing the excited states $D^{*} A$ and $D^{+} A^{-}$. The contribution of $D^{*} A$ will increase with decreasing $E_{D^{*}}-E_{\mathrm{CT}}$, or increasing $V^{*}$. Using experimental data for the TQ1:PCBM system, we estimate $V^{*}$ to be $\sim 6 \mathrm{meV}$. We conclude by discussing the implications for polymer:fullerene photovoltaic devices.

\section{THEORY: A SIMPLE MODEL FOR THE ELECTRONIC STRUCTURE OF THE CT STATE}

Based on previous work on the electronic structure of donor-acceptor interfacial states, ${ }^{1,39,40}$ we adopt a model for which the wave function of the excited interfacial charge transfer state $\Psi_{\mathrm{CT}}$ is considered as a resonance mixture of the wave function of the locally excited donor and/or acceptor $\Psi_{D^{*} A}\left(\Psi_{D A^{*}}\right)$ and the wave function of a fully charge transferred state $\Psi_{D^{+} A^{-}}$:

$$
\Psi_{\mathrm{CT}}=c_{1} \Psi_{D^{+} A^{-}}+c_{2} \Psi_{D^{*} A}+c_{3} \Psi_{D A^{*}},
$$

with $c_{1}^{2}+c_{2}^{2}+c_{3}^{2}=1$. Typically, the prefactors $c_{2}$ and $c_{3}$ are nonzero, but $c_{1} \gg c_{2}$ and $c_{1} \gg c_{3}$. A consequence is that the transition dipole moment between ground state and interfacial state $\vec{M}_{\mathrm{CT}}$ has a component parallel to the transition dipole moment of the donor $\vec{M}_{D^{*} A}$ and the acceptor $\vec{M}_{D A^{*}}$. If the transition dipole of the fully charge transferred state $\vec{M}_{D^{+} A^{-}}$is small, the local excitations $D^{*} A$ and $D A^{*}$ can contribute significantly to $\vec{M}_{\mathrm{CT}}$. This effect is sometimes termed "intensity borrowing" of the CT state from the local excited states on donor or acceptor. ${ }^{39}$ For the films with aligned polymer chains studied in this work, we therefore consider the transition dipole moment of the CT state to consist out of two components [Fig. 6(a)]

$$
\vec{M}_{\mathrm{CT}}=\vec{M}_{\mathrm{CT}}^{\|}+\vec{M}_{\mathrm{CT}}^{r}
$$

Hereby is $\vec{M}_{\mathrm{CT}}^{\|}$in the direction of alignment or direction of the polymer transition dipole moment $\vec{M}_{D^{*} A}$ [Fig. 6(a)]. The second term of Eq. (2), i.e., $\vec{M}_{\mathrm{CT}}^{r}$, represents the contributions of $\Psi_{D^{+} A^{-}}$and $\Psi_{D A^{*}}$ to the overall transition dipole moment of the CT state. Considering the wave-function mixing as a first-order perturbation, one finds that ${ }^{1,39,40}$

$$
\vec{M}_{\mathrm{CT}}^{\|} \approx \frac{V^{*}}{E_{D^{*}}-E_{\mathrm{CT}}} \vec{M}_{D^{*} A} .
$$

Herein represents $V^{*}$ the electronic coupling matrix element between the electronic states $\Psi_{D^{*} A}$ and $\Psi_{D^{+} A^{-}} .\left(V^{*}\right)^{2}$ is proportional to the rate of electron transfer from donor to acceptor. ${ }^{32}$ Theoretical calculations for oligothiophene: $\mathrm{C}_{60}$ systems predict $V^{*}$ to be in the order of 5-50 meV, depending on the molecular conformation. ${ }^{41,42}$ Equation (3) predicts that intensity borrowing becomes more pronounced if $E_{\mathrm{CT}}$ approaches $E_{D^{*}}$. 
In the following paragraphs, we aim to experimentally verify the validity of this model and to determine the relative contributions of $\vec{M}_{\mathrm{CT}}^{\|}$and $\vec{M}_{\mathrm{CT}}^{r}$ to $\vec{M}_{\mathrm{CT}}$ for a few polymer:fullerene material systems. We will achieve this with the aid of polarization anisotropy measurements on mechanically rubbed polymer:fullerene films with aligned polymer transition dipole moments $\vec{M}_{D^{*} A}$.

\section{EXPERIMENTAL RESULTS}

\section{A. Photoluminescence anisotropy}

Films of low molecular weight TQ1 blended with the fullerene derivatives PCBM, bisPCBM, and HG2-V2, in a 4:1 polymer:fullerene weight ratio, were spin cast on $\mathrm{Si}$ substrates. We chose fullerene acceptors with different LUMO levels, aiming to systematically vary $E_{D^{*}}-E_{\mathrm{CT}}$.To improve the adherence of the film, preventing it to come off the substrate during the alignment procedure, a thin interlayer of PEDOT:PSS between substrate and active layer was used. The pure and blend films were aligned by manually rubbing them against a velvet cloth on a hotplate at an elevated temperature $\left(80{ }^{\circ} \mathrm{C}\right.$ or $\left.110^{\circ} \mathrm{C}\right)$. The use of an excess of polymer in a $4: 1$ ratio with the fullerenes is required to obtain a sufficiently high degree of polymer alignment by this mechanical rubbing method.

The Photoluminescence (PL) experiments were performed with the 405-nm excitation laser polarized in the direction of polymer chain alignment. The light emitted by the aligned filmed passes through a Glan-Thompson polarizer with orientation parallel or perpendicular to the direction of alignment. Subsequently, it is collected by a 1-m-long optical liquid light guide, which completely depolarizes the light before it reaches the gratings in the monochromator used to obtain the spectrum. Increasing the integration times of the light-detecting Si CCD array to $10-20 \mathrm{~s}$ increases the sensitivity enabling detection of the considerably quenched emission of the polymer:fullerene blends. A schematic representation of the experiment is shown in Fig. 1(a). The spectra of the emitted light, with polarization parallel $(\|)$ and perpendicular $(\perp)$ to the alignment direction, are shown in Fig. 2 for the pure and blend films aligned at $80{ }^{\circ} \mathrm{C}$.

As compared to pure TQ1, the PL of the blends is considerably weaker. In order to detect the remaining PL of the blends, we have to increase the integration time of the Si CCD detector array by about 100 times, indicating 99\% quenching of the pure polymer emission. We observe residual emission of TQ1, centered at $1.7 \mathrm{eV}$ and an additional, red-shifted emission band. The extent of the red-shift depends on the ionization potential (IP) of the acceptor $\left(\mathrm{IP}_{\text {bisPCBM }}>\mathrm{IP}_{\mathrm{PCBM}}>\right.$ $\left.\mathrm{IP}_{\mathrm{HG} 2-\mathrm{V} 2}\right)$. We therefore assign this new emission feature to originate from the interfacial TQ1:fullerene CT state. Such CT emission bands have been detected previously for several polymer:fullerene systems, using PL and electroluminescence measurements. ${ }^{9} 10,43-45$

The polarization anisotropy $r_{\mathrm{PL}}(E)$ spectra for films aligned at $80{ }^{\circ} \mathrm{C}$ (Fig. 2) and $110{ }^{\circ} \mathrm{C}$ are shown in Fig. 3. $r_{\mathrm{PL}}(E)$ is defined as ${ }^{46}$

$$
r_{\mathrm{PL}}(E)=\frac{\mathrm{PL}_{\|}(E)-\mathrm{PL}_{\perp}(E)}{\mathrm{PL}_{\|}(E)+2 \mathrm{PL}_{\perp}(E)} .
$$

(a)

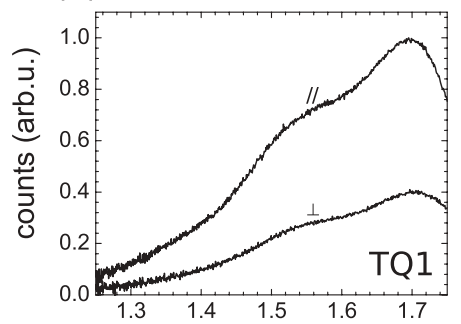

(c)

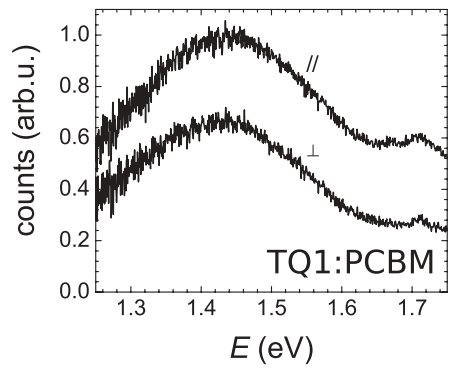

(b)

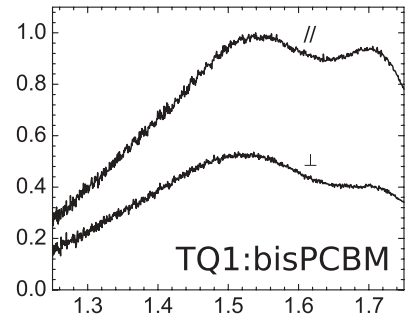

(d)

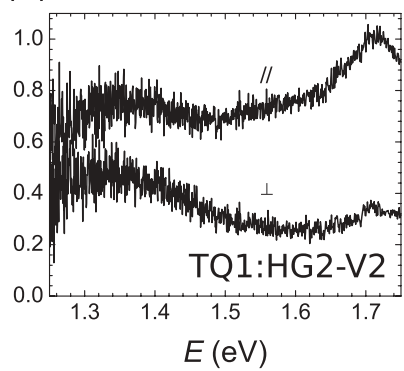

FIG. 2. Normalized PL emission spectra of aligned samples, with the electric field polarized parallel $(\|)$ and perpendicular $(\perp)$ to the rubbing direction. Spectra are shown for (a) pure TQ1, (b) TQ1:bisPCBM, (c) TQ1:PCBM, (d) TQ1:HG2-V2, rubbed at $80{ }^{\circ} \mathrm{C}$. Pure TQ1 emission peaks at $1.7 \mathrm{eV}$. CT emission in the region $1.3-1.6 \mathrm{eV}$ can be detected upon the addition of the fullerene acceptor, red-shifting with increasing acceptor strength.

For an isotropic film, $r_{\mathrm{PL}}(E)$ equals 0 . Values of $r_{\mathrm{PL}}$ are in the interval $[0,1]$ if $\mathrm{PL}_{\|}>P L_{\perp}$ and in $[-0.5,0]$ if $\mathrm{PL}_{\perp}>\mathrm{PL}_{\|}$. For all TQ1-based films, aligned at $80{ }^{\circ} \mathrm{C}$ and $110^{\circ} \mathrm{C}$, and over all emitted photon energies, we find overall positive values of $r_{\mathrm{PL}}(E)$. For the pure TQ1 sample, $r_{\mathrm{PL}}(E)$ is almost constant over the spectral region of strong emission. However, for the blends, $r_{\mathrm{PL}}(E)$ is larger in the spectral region of
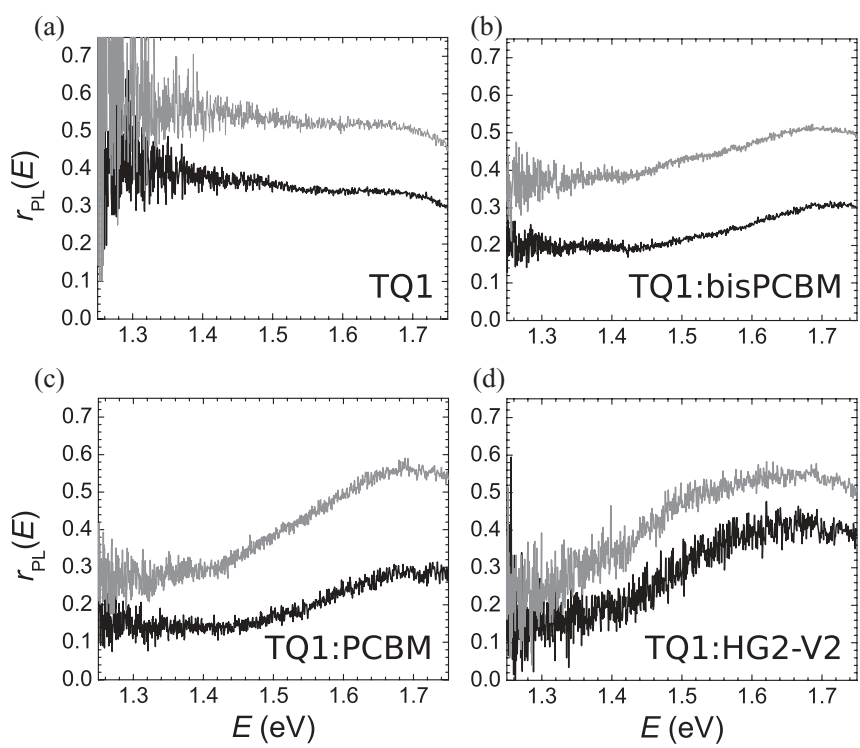

FIG. 3. $r_{\mathrm{PL}}(E)$, calculated via Eq. (4), for (a) pure TQ1, (b) TQ1:bisPCBM, (c) TQ1:PCBM, (d) TQ1:HG2-V2, rubbed at $80{ }^{\circ} \mathrm{C}$ (black) and $110^{\circ} \mathrm{C}$ (gray). $r_{\mathrm{PL}}(E)$ is lower in the spectral region of CT emission than in the spectral region of pure TQ1 emission, but still positive. 
pure TQ1 emission than in the region of CT emission. The CT state emission is hence less polarized than the polymer emission, but nevertheless clearly influenced by the polymer polarization direction. This indicates partial alignment of the CT state transition dipole moment in the direction of polymer alignment (discussion below). Alignment at $110{ }^{\circ} \mathrm{C}$ results in higher $r_{\mathrm{PL}}(E)$ values, both for pure TQ1 emission as for $\mathrm{CT}$ emission. Rubbing alignment at temperatures higher than $110{ }^{\circ} \mathrm{C}$, resulted in a strong increase in pure TQ1 emission, due to extensive phase separation, masking CT emission bands. We also attempted PL experiments on rubbing aligned P3HT:fullerene blends. However, due to the larger phase separation in those material systems, CT emission could not be detected. CT absorption on the other hand could be detected for both P3HT:fullerene and TQ1:fullerene systems, and is the subject of the following paragraph.

\section{B. Photocurrent anisotropy}

Photovoltaic devices comprising rubbing aligned active layers of TQ1:fullerene blends, all in 4:1 ratio and P3HT:fullerene blends in a 2:1 ratio, were prepared in the standard architecture using PEDOT:PSS coated ITO as substrate. The active layers were mechanically rubbed with a velvet cloth prior to $\mathrm{LiF} / \mathrm{Al}$ cathode deposition. Rubbing alignment was done on blends with excess polymer and at $140^{\circ} \mathrm{C}$ and $130{ }^{\circ} \mathrm{C}$ for the TQ1:fullerene and P3HT:fullerene blends, respectively, in order to achieve large anisotropy values in the region of polymer absorption. The resulting photovoltaic devices delivered a sufficient amount of photocurrent at short circuit in order to perform highly sensitive measurements by Fouriertransform photocurrent spectroscopy (FTPS). ${ }^{47}$ Briefly, FTPS uses the external light beam of an FTIR with external beam output option, equipped with a halogen lamp and quartz beam splitter in order to obtain spectra in the visible and near-infrared spectral region. The FTIRs modulated output light beam illuminates the organic photovoltaic devices under investigation. The photocurrent signal produced by the shorted photovoltaic device is amplified and fed back into the FTIR, where analog to digital and Fourier transformation of the signal occurs, yielding the photocurrent spectrum. The direction of polarization of the light incident on the photovoltaic device is selected with a Glan-Thompson polarizer. A schematic representation is shown in Fig. 1(b). Throughout this paper, we present the obtained spectra as external quantum efficiency (EQE). The EQE is defined as the number of electrons flowing through the short-circuited electrodes, per incident photon.

In Figs. 4(a), 4(c), 4(e) and 5(a), 5(c), 5(e), EQE spectra with light polarized parallel $(\|)$ and perpendicular $(\perp)$ to the direction of polymer chain alignment are shown, respectively, for the TQ1:fullerene photovoltaic devices and for the P3HT:fullerene photovoltaic devices.

For TQ1 blended with all three acceptors, a weak absorption shoulder indicative of CT absorption can be detected in the region $<1.6 \mathrm{eV}$. This absorption band shifts to lower photon energies, similar to the CT emission band, revealed in the PL experiments above. For further interpretation of these measurements, we define the polarization anisotropy $r_{\mathrm{EQE}}(E)$, similar to the polarization anisotropy for emission in Eq. (4),
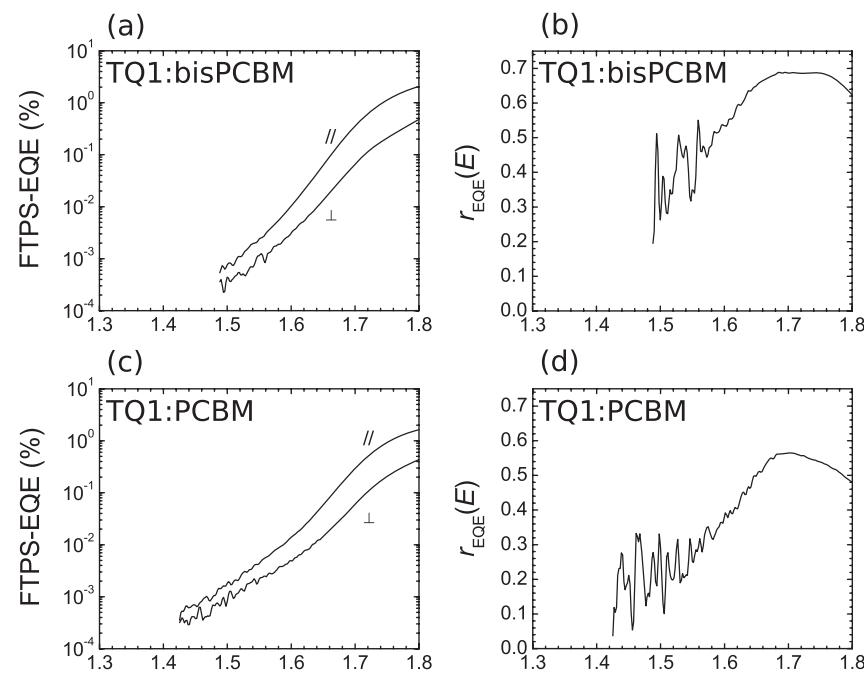

(d)
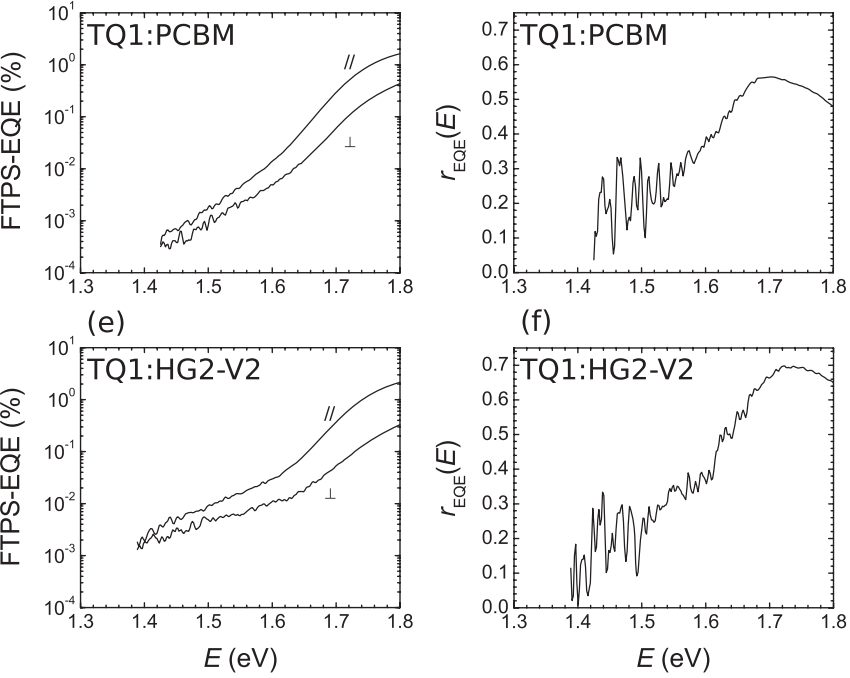

(f)

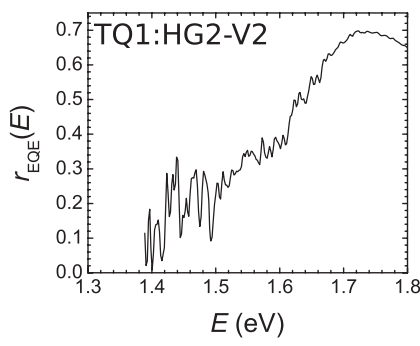

FIG. 4. EQE spectra measured by FTPS with the polarization of the incident light parallel $(\|)$ and perpendicular $(\perp)$ to the direction of rubbing alignment. Spectra are shown for photovoltaic devices comprising active layers of (a) TQ1:bisPCBM (4:1), (c) TQ1:PCBM (4:1), (e) TQ1:HG2-V2 (4:1), aligned at $140{ }^{\circ} \mathrm{C} . r_{\mathrm{EQE}}(E)$ for the corresponding blends is shown in, respectively, (b), (d), and (f).

as

$$
r_{\mathrm{EQE}}(E)=\frac{\mathrm{EQE}_{\|}(E)-\mathrm{EQE}_{\perp}(E)}{\mathrm{EQE}_{\|}(E)+2 \mathrm{EQE}_{\perp}(E)} .
$$

For the TQ1:fullerene systems, $r_{\mathrm{EQE}}(E)$ is shown in Figs 4(b), $4(\mathrm{~d})$, and $4(\mathrm{f}) . r_{\mathrm{EQE}}(E)$ peaks in the region of weak polymer absorption $(\sim 1.7 \mathrm{eV})$. For higher photon energies, $r_{\mathrm{EQE}}(E)$ decreases because the samples become optically thick. In the spectral region of polymer absorption but where the sample is optically thin, $r_{\mathrm{EQE}}(E)$ exhibits a maximum. In the region of CT absorption, $r_{\mathrm{EQE}}(E)$ is lower as compared to pure polymer absorption but remains larger than zero, consistent with the PL experiments.

For the P3HT:fullerene devices, $r_{\mathrm{EQE}}(E)$ spectra are shown in Figs. 5(b), 5(d), and 5(f). We observe rich spectra with varying regions of anisotropy. At high photon energies $(\sim 1.9 \mathrm{eV})$, P3HT absorption with a high anisotropy dominates the spectrum. $r_{\mathrm{EOE}}(E)$ changes sharply in the region from 1.75 to $1.9 \mathrm{eV}$ and has its lowest value at $1.75 \mathrm{eV}$, where fullerene absorption dominates. Below $1.7 \mathrm{eV}$, in the spectral region where $\mathrm{CT}$ absorption dominates, $r_{\mathrm{EQE}}(E)$ has its highest value in its lowest energy part. The spectral position of the low-energy band with high $r_{\mathrm{EOE}}(E)$ shifts to lower energies with increasing acceptor strength. These different features within the CT band indicate that two types of CT states are present in P3HT:fullerene blends: We attribute the low 
(a)
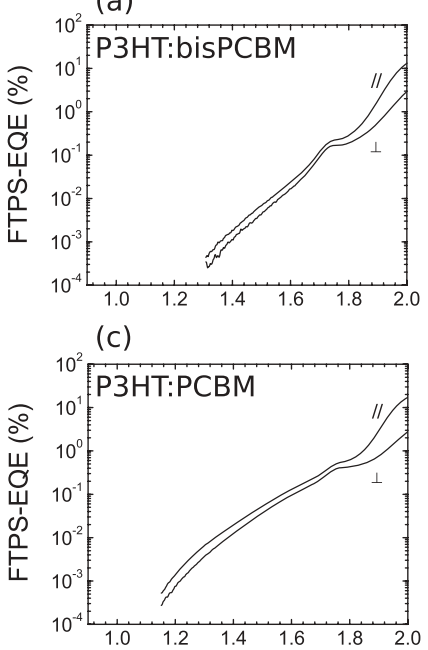

(e)

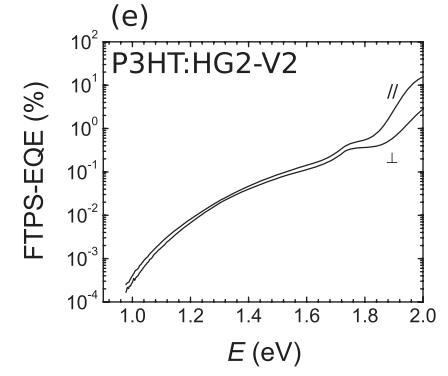

FIG. 5. EQE spectra measured by FTPS with the polarization of the incident light parallel $(\|)$ and perpendicular $(\perp)$ to the direction of rubbing alignment. Spectra are shown for photovoltaic devices comprising active layers of (a) P3HT:bisPCBM (2:1), (c) P3HT:PCBM (2:1), (e) P3HT:HG2-V2 (2:1), aligned at $130{ }^{\circ} \mathrm{C}$. $r_{\mathrm{EQE}}(E)$ for the corresponding blends is shown in, respectively, (b), (d), and (f).

$r_{\mathrm{EQE}}(E)$, high photon energy (but $<1.7 \mathrm{eV}$ ) spectral part to CT absorption involving fullerene and poorly aligned, amorphous $\mathrm{P} 3 \mathrm{HT}$. The lower energy, higher $r_{\mathrm{EQE}}(E)$ part is attributed to CT absorption involving fullerene and well-aligned, crystalline P3HT. This assignment is confirmed by previous experiments: The presence and spectral position of the low-energy part of the CT band is strongly affected by regioregularity and the degree of P3HT crystallization. ${ }^{19,34}$

\section{DISCUSSION}

\section{A. Assignment of the CT absorption and emission anisotropy to alignment of the transition dipole moment}

A general conclusion which can be made for the studied TQ1:fullerene and P3HT:fullerene systems is that the polarization anisotropies larger than zero are observed in the spectral region of CT absorption and emission. However, the anisotropy in the CT band is substantially lower than the anisotropy of the pure polymer excited state. We will argue that this is due to partial alignment of the $\mathrm{CT}$ transition dipole moment in the direction of the polymer chains. But, before making this attribution, we must first rule out the influence of the alignment induced anisotropy of the index of refraction.

The indexes of refraction for light polarized parallel $\left(n_{\|}\right)$ and perpendicular $\left(n_{\perp}\right)$ to the polymer chains are different, and can vary considerably. $n_{\|}-n_{\perp}$ can reach up to $0.7,{ }^{48}$ for strongly aligned films. Assuming $n_{\|}-n_{\perp}=0.7$ with and average refractive index of 2.0, typical for the subgap region of conjugated polymers, ${ }^{48}$ this will result in a maximum difference in transmittance for parallel and perpendicular polarization at the film-air interface of $\sim 10 \%$ and even less at the film-glass interface. In both the photoluminescence and FTPS measurements, the observed dichroic ratios are sufficiently high to rule out substantial effects of this index of refraction difference on the experimentally obtained polarization anisotropies. We therefore attribute the observed anisotropy of both polymer and CT transitions to an alignment of transition dipole moments of polymer excited states and CT excited states. As shown in the following section, this provides information about the actual electronic structure of the CT states.

\section{B. Contribution of intensity borrowing versus $E_{D^{*}}-E_{C T}$}

In this section, we aim to experimentally determine the relative contribution of $\vec{M}_{\mathrm{CT}}^{\|}$and $\vec{M}_{\mathrm{CT}}^{r}$ to $\vec{M}_{\mathrm{CT}}$ with the aid of the polarization anisotropy data obtained via FTPS and PL measurements on aligned films, discussed above. In order to do so, we first have to derive a relation between $r_{\mathrm{PL}}(E)\left[r_{\mathrm{EQE}}(E)\right]$ in the region of donor emission (absorption) and CT emission (absorption) and the magnitudes of the involved vectors $\left|\vec{M}_{\mathrm{CT}}^{\|}\right|$ and $\left|\vec{M}_{\mathrm{CT}}^{r}\right|$.

The angular distribution of $\vec{M}_{D^{*} A}$ can be obtained from the anisotropy $r_{\mathrm{PL}}(E)\left[r_{\mathrm{EQE}}(E)\right]$ in the region of polymer absorption/emission. The direction of $\vec{M}_{\mathrm{CT}}^{r}$ is determined by the directions of the purely ionic transition dipole moment and of the fullerene transition dipole moments, which we assume to be unaffected by the rubbing alignment process. We therefore will assume $\vec{M}_{\mathrm{CT}}^{r}$ to have a random orientation (see Fig. 6). Using these assumptions, we can calculate the ratio $\frac{\left|M_{\mathrm{CT}}^{r}\right|}{\left|M_{\mathrm{CT}}^{\|}\right|}$from the ratio between the anisotropy values $r_{\mathrm{PL}}(E)\left[r_{\mathrm{EQE}}(E)\right]$ in the CT spectral region $\left(r_{\mathrm{CT}}\right)$ and donor spectral region $\left(r_{D^{*}}\right)$ (see the derivation in the Appendix):

$$
\frac{\left|\vec{M}_{\mathrm{CT}}^{r}\right|}{\left|\vec{M}_{\mathrm{CT}}^{\|}\right|}=\frac{1}{2 y}\left(1-y+\sqrt{2 y-3 y^{2}+1}\right)
$$

with $y=\frac{r_{\mathrm{CT}}}{r_{D^{*}}}$. Figure 6 shows the obtained $\frac{\left|\vec{M}_{\mathrm{CT}}^{r}\right|}{\left|\vec{M}_{\mathrm{CT}}^{\|}\right|}$ratio as a function of $E_{D^{*}}-E_{\mathrm{CT}}$. It was determined from the $r_{\mathrm{PL}}(E)$ spectra for rubbing aligned films of TQ1, blended with bisPCBM, $\mathrm{PC}_{61} \mathrm{BM}, \mathrm{PC}_{71} \mathrm{BM}$, and $\mathrm{HG} 2-\mathrm{V} 2$. Additionally, we used $r_{\mathrm{EQE}}(E)$ to determine $\frac{\left|\vec{M}_{\mathrm{CT}}^{r}\right|}{\left|\vec{M}_{\mathrm{CT}}^{\|}\right|}$for both TQ1 and P3HT, blended with bisPCBM, PCBM, and HG2-V2. For all these material systems, $E_{D^{*}}-E_{\mathrm{CT}}$ was determined from the PL and/or FTPS spectra.

Obtained $\frac{\left|\vec{M}_{\mathrm{CT}}^{r}\right|}{\left|\vec{M}_{\mathrm{CT}}^{\|}\right|}$ratios are in the range of 1.0 to 5.0, indicating that the CT state borrows a substantial amount of intensity from the donor excitation. Moreover, for a given donor polymer, $\frac{\left|\vec{M}_{\mathrm{CT}}^{r}\right|}{\left|\vec{M}_{\mathrm{CT}}^{\|}\right|}$decreases for decreasing $E_{D^{*}}-E_{\mathrm{CT}}$. Using Eq. (3), we 
(a)

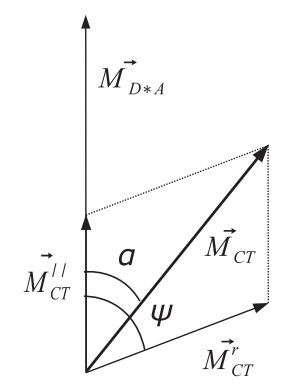

(b)

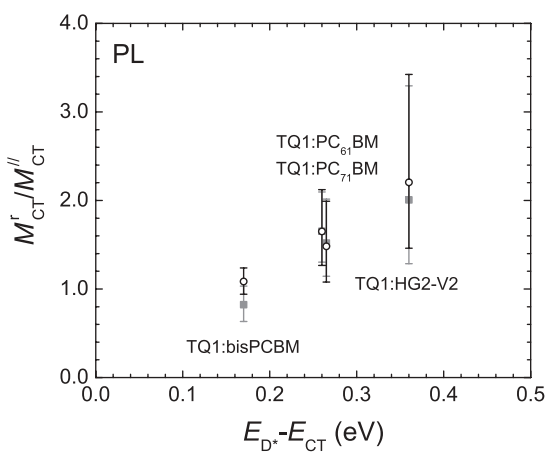

(c)

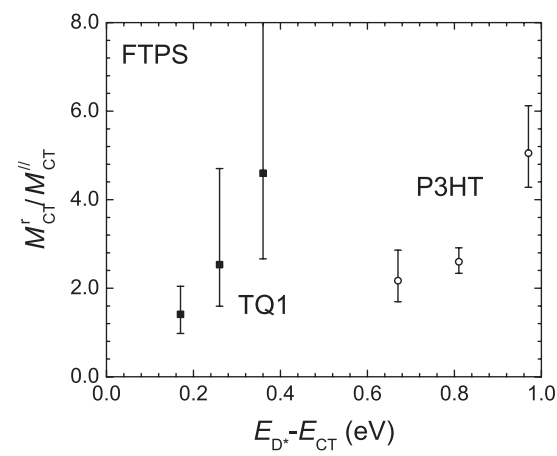

FIG. 6. (a) The transition dipole moment of the CT state $\vec{M}_{\mathrm{CT}}$ as the sum of a component $\vec{M}_{\mathrm{CT}}^{\|}$, parallel to the transition dipole moment of the donor excitation $\vec{M}_{D^{*} A}$ and a component $\vec{M}_{\mathrm{CT}}^{r}$, assumed to be randomly oriented. The ratio between the magnitudes of these components $\frac{\mid \vec{M}_{\mathrm{CT}}}{\left|\vec{M}_{\mathrm{CT}}^{r}\right|}$ is calculated from the anisotropies in the spectral region of CT emission/absorption using Eq. (6). $\frac{\left|\vec{M}_{\mathrm{CT}}^{r}\right|}{\left|\vec{M}_{\mathrm{CT}}^{r}\right|}$ is shown as a function of $E_{D^{*}}-\vec{E}_{\mathrm{CT}}$ in (b), calculated from $r_{\mathrm{PL}}(E)$ of TQ1:fullerene blends aligned at $80{ }^{\circ} \mathrm{C}$ (filled squares) and $110^{\circ} \mathrm{C}$ (open circles). In (c), $\frac{\left|M_{\mathrm{CT}}\right|}{\left|\bar{M}_{\mathrm{CT}}^{r}\right|}$ as a function of $E_{D^{*}}-E_{\mathrm{CT}}$ is shown for the TQ1 (filled squares) and $\mathrm{P} 3 \mathrm{HT}$ (open circles) based systems, calculated from $r_{E Q E}(E)$.

indeed predict a linear relation between $\frac{\left|\vec{M}_{\mathrm{CT}}^{r}\right|}{\left|\vec{M}_{\mathrm{CT}}^{\mathrm{a}}\right|}$ and $E_{D^{*}}-E_{\mathrm{CT}}$ :

$$
\frac{\left|\vec{M}_{\mathrm{CT}}^{r}\right|}{\left|\vec{M}_{\mathrm{CT}}^{\|}\right|} \approx \frac{\left|\vec{M}_{\mathrm{CT}}^{r}\right|}{V^{*}\left|\vec{M}_{D^{*} A}\right|}\left(E_{D^{*}}-E_{\mathrm{CT}}\right) .
$$

The observed linear relation indicates that the prefactor $\frac{\left|\vec{M}_{\mathrm{CT}}^{r}\right|}{V^{*}\left|\vec{M}_{D^{*} A}\right|}$ does not depend strongly on the fullerene acceptor used. It does, however, depend on the donor material (TQ1 or $\mathrm{P} 3 \mathrm{HT}$ ) used. Additionally, we find a twofold difference in prefactor, whether using the PL or FTPS data for the TQ1:fullerene systems. Assuming $M_{\mathrm{CT}}^{r}$ is the same for both measurements, this indicates that the CT emission observed in PL originate from CT states with an electronic coupling $V^{*}$ twice as large as the CT states observed in the FTPS measurements. This is consistent with the fact that PL experiments probe those DA interfaces with the highest likelihood to emit light and thus the lowest likelihood to produce photocurrent. However, the photocurrent measured by FTPS originates primarily from those DA interfaces which efficiently dissociate. PL and FTPS therefore seem to probe different CT state conformations, both present in disordered blends. A similar reasoning was used to explain the difference between the CT emission spectrum as measured by PL and electroluminescence. ${ }^{10}$

\section{Estimation of $V^{*}$}

In this section, we estimate $V^{*}$ for the TQ1:PCBM material system by comparing the extinction coefficients of the donor optical transition $\varepsilon_{D^{*}}$ and CT optical transition $\varepsilon_{\mathrm{CT}} \cdot \varepsilon_{D^{*}}$ and $\varepsilon_{\mathrm{CT}}$ are proportional to the square of their respective transition dipole moments. From Eqs. (2) and (3) we can determine the ratio between these two extinction coefficients:

$$
\frac{\varepsilon_{\mathrm{CT}}}{\varepsilon_{D^{*}}} \approx\left(\frac{V^{*}}{E_{D^{*}}-E_{\mathrm{CT}}}\right)^{2}\left(1+\frac{\left|\vec{M}_{\mathrm{CT}}^{r}\right|^{2}}{\left|\vec{M}_{\mathrm{CT}}^{\|}\right|^{2}}\right) .
$$

For the TQ1:PCBM system, we obtain $E_{D^{*}}-E_{\mathrm{CT}}=0.26 \mathrm{eV}$ and $\frac{\left|\vec{M}_{\mathrm{CT}}^{r}\right|}{\left|\vec{M}_{\mathrm{CT}}^{\|}\right|}=2.5$ from the FTPS measurements (Fig. 6). In order to estimate $\frac{\varepsilon_{\mathrm{CT}}}{\varepsilon_{D^{*}}}$, we use a TQ1:PCBM blend in 5:95 wt:wt ratio.
Such low amount of TQ1 diluted in PCBM allows us to assume that all conjugated TQ1 segments are in close proximity to a fullerene. In that case, the number of charge transfer complex (CTC) ground states is approximately equal to the number of polymer ground states. The FTPS spectrum of such a blend is shown in Fig. 7. Since the device is optically thin, we can estimate an order of magnitude for the ratio between polymer absorption and CT absorption, hereby neglecting interference effects and assuming that the internal quantum efficiency for excitation in the polymer absorption and CT absorption bands are approximately equal, as is the case for the P3HT:PCBM and MDMO-PPV:PCBM blends. ${ }^{28}$ We estimate $\frac{\varepsilon_{\mathrm{CT}}}{\varepsilon_{D^{*}}}$ by taking the ratio of the integrals of the respective absorption bands. We obtain $\frac{\varepsilon_{\mathrm{CT}}}{\varepsilon_{D^{*}}} \approx 4.10^{-3}$ yielding a value of $6 \mathrm{meV} \pm 3 \mathrm{meV}$ for

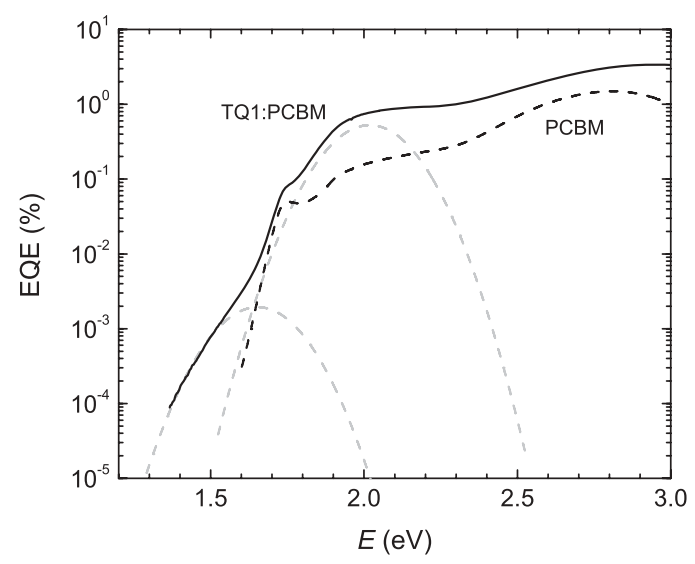

FIG. 7. EQE spectrum for a TQ1:PCBM photovoltaic device with a 5:95 stoichiometry. At such low TQ1 content, it is assumed that all TQ1 chromophores are involved in complexation with PCBM. We reconstruct the EQE spectrum as a linear combination of the EQE spectrum of PCBM (dotted black line) and two parabola for the lowest excited state on the polymer and the CT state, respectively (dotted gray lines). The ratio between oscillator strengths of CT state and lowest TQ1 excitation is estimated as the ratios between the areas under the fitted parabolas. 


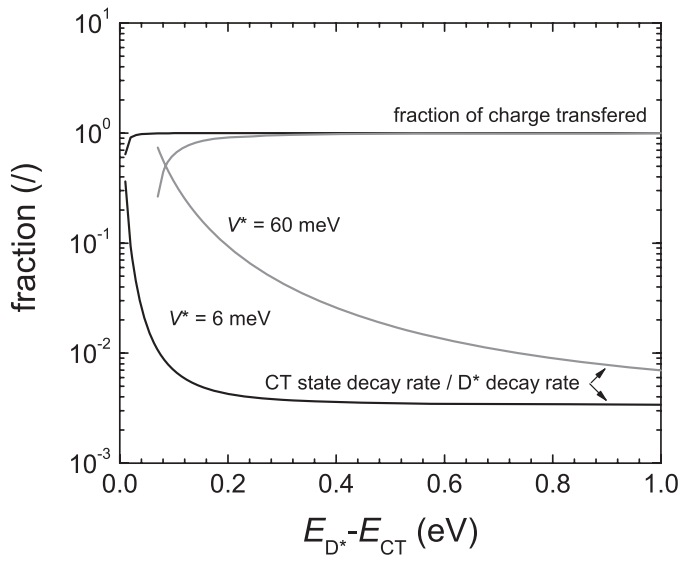

FIG. 8. The fraction of charge transferred $f_{\mathrm{CT}}$ and ratio between the radiative decay rate of the CT state and decay rate of the $D^{*}$ exciton, as a function of $E_{D^{*}}-E_{\mathrm{CT}}$. Curves, given by Eq. (9), are plotted for $V^{*}=6 \mathrm{meV}$ (black) and $V^{*}=60 \mathrm{meV}$ (gray).

$V^{*}$. This experimentally obtained value is in the range of values calculated for other donor-fullerene material systems. ${ }^{41,42}$

\section{IMPLICATIONS FOR POLYMER:FULLERENE PHOTOVOLTAICS AND CONCLUSION}

The above analysis indicates that for TQ1:fullerene and P3HT:fullerene CT states, intensity borrowing from the polymer excited state is significant. Moreover, the contribution of the donor excitation to the CT state increases with decreasing $E_{D^{*}}-E_{\mathrm{CT}}$. Material systems with low $E_{D^{*}}-E_{\mathrm{CT}}$ are of interest for photovoltaic applications since they will have closer to optimum open-circuit voltages. However, this will have consequences relevant for free charge carrier generation in organic donor-acceptor solar cells: upon decreasing $E_{D^{*}}-$ $E_{\mathrm{CT}}$, an increase of radiative CT state decay rate will occur, as well as a decrease in the fraction of charge transferred from donor to acceptor. Both will now be discussed shortly.

The radiative decay rate of the $\mathrm{CT}$ state is given by the square of the transition dipole moment:

$$
\left|\vec{M}_{\mathrm{CT}}\right|^{2} \approx\left(\frac{V^{*}}{E_{D^{*}}-E_{\mathrm{CT}}}\right)^{2}\left|\vec{M}_{D^{*} A}\right|^{2}+\left|\vec{M}_{\mathrm{CT}}^{r}\right|^{2} .
$$

The fraction of charge transferred $\left(f_{\mathrm{CT}}\right)$ is given by $1,39,40$

$$
f_{\mathrm{CT}}=1-\left(\frac{V^{*}}{E_{D^{*}}-E_{\mathrm{CT}}}\right)^{2}
$$

with $E_{D^{*}}-E_{\mathrm{CT}} \gg V^{*}$ for this approximation to be valid. Plots of $\frac{\left|\vec{M}_{\mathrm{CT}}\right|^{2}}{\left|\vec{M}_{D^{*} A}\right|^{2}}$ and $f_{\mathrm{CT}}$ as a function of $E_{D^{*}}-E_{\mathrm{CT}}$ are shown in Fig. 8 for $V^{*}=6 \mathrm{meV}$ and $\frac{\left|\vec{M}_{\mathrm{CT}}^{r}\right|^{2}}{\left|\vec{M}_{D^{*}}\right|^{2}}$ determined for the TQ1:PCBM material system above. We also plot curves for a tenfold higher value of $V^{*}=60 \mathrm{meV}$. For $V^{*}=6 \mathrm{meV}$, Fig. 8 shows that, when decreasing $E_{D^{*}}-E_{\mathrm{CT}}$ to values smaller than $\sim 0.1 \mathrm{eV}$, the radiative decay rate of the CT state starts to increase. For very small values of $E_{D^{*}}-E_{\mathrm{CT}}$, the intensity borrowing effect becomes very significant and the radiative decay rate of the $\mathrm{CT}$ states becomes similar to the radiative decay rate of the pure donor. A tenfold increase of the coupling $\left(V^{*}=60 \mathrm{meV}\right)$ increases the radiative decay rate of the CT state significantly. For such values of $V^{*}$, intensity borrowing starts having a significant effect already for $E_{D^{*}}-E_{\mathrm{CT}}$ differences smaller than $\sim 0.2 \mathrm{eV}$.

The free charge carrier generation process in polymer:fullerene solar cells is a competition between the decay of the CT state to the ground state and dissociation into free carriers. Decreasing $E_{D^{*}}-E_{\mathrm{CT}}$ in order to optimize the photovoltage increases the (radiative) decay rate of the CT state. For low values of $E_{D^{*}}-E_{\mathrm{CT}}$, the CT state decay rate might be increased to such high values that it efficiently competes with the dissociation rate. This will result in less free charge carriers generated and constitutes a loss in photocurrent.

Recently, Street ${ }^{49}$ used a slightly different approach to predict an increase in radiative CT state decay rate upon decreasing the donor and acceptor band offset, due to an increased wave-function overlap between the charge carrier in the highest occupied molecular orbital (HOMO) of the donor and the LUMO of the acceptor. A larger electronic coupling intensifies this effect. In fact, the larger electronic coupling of the oligothiophene:DPI CT state as compared to the oligothiophene: $\mathrm{C}_{60} \mathrm{CT}$ state has been proposed to be a reason for a decreased photocurrent generation in P3HT:DPI photovoltaic devices as compared to P3HT: PCBM. $^{41}$

Figure 8 also shows that, for a sufficiently large $V^{*}$, a second consequence of a reduced $E_{D^{*}}-E_{\mathrm{CT}}$ offset is that the fraction of charge transferred in the CT state can become considerably less than 1. Such an effect is expected to decrease the rate of CT state dissociation into free charge carriers, again having a negative effect on photocurrent generation.

Experimental observations indicate that charge generation indeed seems to be affected by the $E_{D^{*}}-E_{\mathrm{CT}}$ difference, although molecular order at the interface has been shown to also play a role. ${ }^{14,26,27}$ Previously proposed loss mechanisms for such low "driving force" systems are repopulation of the donor singlet state and the involvement of triplet states. Triplets on the donor polymer can be populated when their energy is lower than $E_{\mathrm{CT}}$. For polymer:polymer systems, this has been shown to be a loss mechanism, limiting the photocurrent. ${ }^{20,24}$ However, when charge transfer and the subsequent dissociation of CT states into free charge carriers is faster than intersystem crossing, this does not constitute a major loss mechanism. ${ }^{23}$ The intensity borrowing mechanism proposed in this paper should be considered as another possible loss mechanism limiting the photocurrent for small "driving force" polymer:fullerene systems. This loss mechanism, however, can be reduced by reducing the electronic coupling matrix element $V^{*}$ by, for example, increasing the distance between donor and acceptor molecule. However, decreasing $V^{*}$ will unavoidably result in a decrease in electron transfer rate, which is proportional to $\left(V^{*}\right)^{2} .^{32}$

The analysis presented above allows experimental determination of $V^{*}$, which we find is $\sim 6 \mathrm{meV}$ for the TQ1:PCBM system studied in this work. Assuming that this is a typical value for polymer:fullerene systems, a lower limit for the ratio between the molar extinction coefficient of CT absorption and pure polymer absorption can be calculated: Assuming intensity borrowing as the only cause for CT absorption, a ratio $\alpha_{\mathrm{CT}} / \alpha D *=\frac{\left(V^{*}\right)^{2}}{\left(E_{D^{*}}-E_{\mathrm{CT}}\right)^{2}}$ between $10^{-2}$ and $10^{-4}$ can be 
calculated for $E_{D^{*}}-E_{\mathrm{CT}}$ between 0.06 and $0.6 \mathrm{eV}$, typical energy offsets for organic photovoltaic applications. For finely intermixed systems, given a sufficiently high sensitive technique, this means that $\mathrm{CT}$ absorption bands will always be detectable if $E_{\mathrm{CT}}$ is lower than $E_{D^{*}}$ and $V^{*}$ is sufficiently large.

\section{ACKNOWLEDGMENTS}

We gratefully acknowledge funding from the Swedish Energy Agency through the program Tandem, as well as the Swedish Research Council (VR), VINNOVA and the Knut and Alice Wallenberg foundation for providing equipment financing. Solenne BV is thanked for providing HG2-V2 used in this work.

\section{APPENDIX: RELATION BETWEEN $\frac{r_{C T}}{r_{D^{*}}}$ and $\frac{\left|\vec{M}_{C T}^{r}\right|}{\left|\vec{M}_{\mathrm{CT}}^{\|}\right|}$}

Define $\theta_{D^{*}}$ and $\theta_{\mathrm{CT}}$ as the angle between the direction of alignment and $\vec{M}_{D^{*} A}$ and $\vec{M}_{\mathrm{CT}}$, respectively. $\alpha$ is the angle between $\vec{M}_{\mathrm{CT}}$ and $\vec{M}_{D^{*} A}$. If we consider the direction of alignment a symmetry axis, the following relation is valid ${ }^{46}$ :

$$
\begin{aligned}
\overline{\left|\vec{M}_{\mathrm{CT}}\right|^{2} \cos ^{2}\left(\theta_{\mathrm{CT}}\right)}= & \overline{\left|\vec{M}_{\mathrm{CT}}\right|^{2} \cos ^{2}(\alpha)} \overline{\cos ^{2}\left(\theta_{D^{*}}\right)} \\
& +\frac{1}{2} \overline{\left.\vec{M}_{\mathrm{CT}}\right|^{2} \sin ^{2}(\alpha)} \overline{\sin ^{2}\left(\theta_{D^{*}}\right)}
\end{aligned}
$$

with $\bar{x}$ symbolizing the average value of $x$ over a macroscopic fraction of the material. $\vec{M}_{\mathrm{CT}}$ is described by the vector sum given in Eq. (2). Define $\psi$ as the angle between $\vec{M}_{\mathrm{CT}}^{r}$ and $\vec{M}_{D^{*} A}$, then

$$
\begin{gathered}
\left|\vec{M}_{\mathrm{CT}}\right| \cos (\alpha)=\left|\vec{M}_{\mathrm{CT}}^{r}\right| \cos (\psi)+\left|\vec{M}_{\mathrm{CT}}^{\|}\right| \\
\left|\vec{M}_{\mathrm{CT}}\right| \sin (\alpha)=\left|\vec{M}_{\mathrm{CT}}^{r}\right| \sin (\psi) .
\end{gathered}
$$

In this work, we assume that $\psi$ can take any random value between $0^{\circ}$ and $90^{\circ}$, or

$$
\begin{aligned}
& \overline{\cos (\psi)}=\frac{1}{2}, \\
& \overline{\cos ^{2}(\psi)}=\frac{1}{3} .
\end{aligned}
$$

Substituting the equations above in Eq. (A1) gives

$$
\begin{aligned}
\overline{\left|\vec{M}_{\mathrm{CT}}\right|^{2} \cos ^{2}\left(\theta_{\mathrm{CT}}\right)}= & \left(\left|\vec{M}_{\mathrm{CT}}^{r}\right|\left|\vec{M}_{\mathrm{CT}}^{\|}\right|+\left|\vec{M}_{\mathrm{CT}}^{\|}\right|^{2}\right) \overline{\cos ^{2}\left(\theta_{D^{*}}\right)} \\
& +\frac{1}{3}\left|\vec{M}_{\mathrm{CT}}^{r}\right|^{2} .
\end{aligned}
$$

Additionally, from Eq. (2), using Eq. (A4), we get

$$
\overline{\left|\vec{M}_{\mathrm{CT}}\right|^{2}}=\left|\vec{M}_{\mathrm{CT}}^{r}\right|^{2}+\left|\vec{M}_{\mathrm{CT}}^{\|}\right|^{2}+\left|\vec{M}_{\mathrm{CT}}^{r}\right|\left|\vec{M}_{\mathrm{CT}}^{\|}\right| \text {. }
$$

The relations between the angular distribution and the anisotropy values are ${ }^{46}$

$$
\begin{gathered}
\frac{\overline{\left|\vec{M}_{\mathrm{CT}}\right|^{2} \cos ^{2}\left(\theta_{\mathrm{CT}}\right)}}{\overline{\left|\vec{M}_{\mathrm{CT}}\right|^{2}}}=\frac{2 r_{\mathrm{CT}}+1}{3}, \\
\frac{\cos ^{2}\left(\theta_{D^{*}}\right)}{3}=\frac{2 r_{D^{*}}+1}{3} .
\end{gathered}
$$

Using these equations, we get

$$
\frac{r_{\mathrm{CT}}}{r_{D *}}=\frac{\left|\vec{M}_{\mathrm{CT}}^{r}\right|\left|\vec{M}_{\mathrm{CT}}^{\|}\right|+\left|\vec{M}_{\mathrm{CT}}^{\|}\right|^{2}}{\left|\vec{M}_{\mathrm{CT}}^{r}\right|\left|M_{\mathrm{CT}}^{\|}\right|+\left|\vec{M}_{\mathrm{CT}}^{\|}\right|^{2}+\left|\vec{M}_{\mathrm{CT}}^{r}\right|^{2}},
$$

which we can solve in order to obtain an expression for $\frac{\left|\vec{M}_{\mathrm{CT}}^{r}\right|}{\left|\vec{M}_{\mathrm{CT}}^{\|}\right|}$ as a function of $y=\frac{r_{\mathrm{CT}}}{r_{D *}}$ :

$$
\frac{\left|\vec{M}_{\mathrm{CT}}^{r}\right|}{\left|\vec{M}_{\mathrm{CT}}^{\|}\right|}=\frac{1}{2 y}\left(1-y+\sqrt{2 y-3 y^{2}+1}\right) .
$$

*Present address: Department of Materials Science and Engineering, Geballe Laboratory for Advanced Materials, McCullough Building, Stanford University, Stanford, California 94305, United States; vandewal@stanford.edu

${ }^{1}$ Y.-S. Huang, S. Westenhoff, I. Avilov, P. Sreearunothai, J. M. Hodgkiss, C. Deleener, R. H. Friend, and D. Beljonne, nature mater. 7, 483 (2008).

${ }^{2}$ A. Morteani, A. Dhoot, J.-S. Kim, C. Silva, N. Greenham, C. Murphy, E. Moons, S. Cina, J. Burroughes, and R. Friend, Adv. Mater. 15, 1708 (2003).

${ }^{3}$ A. C. Morteani, P. Sreearunothai, L. M. Herz, R. H. Friend, and C. Silva, Phys. Rev. Lett. 92, 247402 (2004).

${ }^{4}$ K. Goushi, K. Yoshida, K. Sato, and C. Adachi, Nature Photon. 6, 253 (2012).

${ }^{5}$ S. H. Park, A. Roy, S. Beaupr, S. Cho, N. Coates, J. S. Moon, D. Moses, M. Leclerc, K. Lee, and A. J. Heeger, nature photon. 3, 297 (2009).

${ }^{6}$ H.-Y. Chen, J. Hou, S. Zhang, Y. Liang, G. Yang, Y. Yang, L. Yu, Y. Wu, and G. Li, nature photon. 3, 649 (2009).

${ }^{7}$ Y. Liang, Z. Xu, J. Xia, S.-T. Tsai, Y. Wu, G. Li, C. Ray, and L. Yu, Adv. Mater. 22, E135 (2010).
${ }^{8}$ H. Kim, J. Y. Kim, S. H. Park, K. Lee, Y. Jin, J. Kim, and H. Suh, Appl. Phys. Lett. 86, 183502 (2005).

${ }^{9}$ K. Tvingstedt, K. Vandewal, A. Gadisa, F. Zhang, J. Manca, and O. Inganas, J. Am. Chem. Soc. 131, 11819 (2009).

${ }^{10} \mathrm{~K}$. Tvingstedt, K. Vandewal, F. Zhang, and O. Inganas, J. Phys. Chem. C 114, 21824 (2010).

${ }^{11}$ M. C. Scharber, C. Lungenschmied, H.-J. Egelhaaf, G. Matt, M. Bednorz, T. Fromherz, J. Gao, D. Jarzab, and M. A. Loi, Energy Environ. Sci. 4, 5077 (2011)

${ }^{12}$ J.-L. Bredas, J. E. Norton, J. Cornil, and V. Coropceanu, Acc. Chem. Res. 42, 1691 (2009).

${ }^{13}$ D. Beljonne, J. Cornil, L. Muccioli, C. Zannoni, J.-L. Bredas, and F. Castet, Chem. Mater. 23, 591 (2011).

${ }^{14}$ C. Deibel, T. Strobel, and V. Dyakonov, Adv. Mater. 22, 4097 (2010).

${ }^{15}$ A. Maurano, R. Hamilton, C. G. Shuttle, A. M. Ballantyne, J. Nelson, B. O'Regan, W. Zhang, I. McCulloch, H. Azimi, M. Morana, C. J. Brabec, and J. R. Durrant, Adv. Mater. 22, 4987 (2010).

${ }^{16}$ W. J. P. Jr., S. Yoo, and B. Kippelen, Appl. Phys. Lett. 93, 193308 (2008). 
${ }^{17}$ K. Vandewal, K. Tvingstedt, A. Gadisa, O. Inganas, and J. V. Manca, nature mater. 8, 904 (2009).

${ }^{18}$ C. W. Schlenker and M. E. Thompson, Chem. Commun. 47, 3702 (2011).

${ }^{19}$ K. Vandewal, A. Gadisa, W. D. Oosterbaan, S. Bertho, F. Banishoeib, I. V. Severen, L. Lutsen, T. J. Cleij, D. Vanderzande, and J. V. Manca, Adv. Funct. Mater. 18, 2064 (2008).

${ }^{20}$ D. Veldman, S. C. J. Meskers, and R. A. J. Janssen, Adv. Funct. Mater. 19, 1939 (2009).

${ }^{21}$ K. Vandewal, K. Tvingstedt, A. Gadisa, O. Inganas, and J. V. Manca, Phys. Rev. B 81, 125204 (2010).

${ }^{22}$ K. Vandewal, Z. Ma, J. Bergqvist, Z. Tang, E. Wang, P. Henriksson, K. Tvingstedt, M. R. Andersson, F. Zhang, and O. Inganas, Adv. Funct. Mater., doi: 10.1002/adfm.201200608.

${ }^{23}$ D. Di Nuzzo, A. Aguirre, M. Shahid, V. S. Gevaerts, S. C. J. Meskers, and R. A. J. Janssen, Adv. Mater. 22, 4321 (2010).

${ }^{24}$ S. Westenhoff, I. A. Howard, J. M. Hodgkiss, K. R. Kirov, H. A. Bronstein, C. K. Williams, N. C. Greenham, and R. H. Friend, J. Am. Chem. Soc. 130, 13653 (2008).

${ }^{25}$ D. H. K. Murthy, M. Gao, M. J. W. Vermeulen, L. D. A. Siebbeles, and T. J. Savenije, J. Phys. Chem. C 116, 9214 (2012).

${ }^{26}$ H. Ohkita, S. Cook, Y. Astuti, W. Duffy, S. Tierney, W. Zhang, M. Heeney, I. McCulloch, J. Nelson, D. D. C. Bradley, and J. R. Durrant, J. Am. Chem. Soc. 130, 3030 (2008).

${ }^{27}$ T. M. Clarke and J. R. Durrant, Chem. Rev. 110, 6736 (2010).

${ }^{28}$ J. Lee, K. Vandewal, S. R. Yost, M. E. Bahlke, L. Goris, M. A. Baldo, J. V. Manca, and T. V. Voorhis, J. Am. Chem. Soc. 132, 11878 (2010)

${ }^{29}$ A. A. Bakulin, A. Rao, V. G. Pavelyev, P. H. M. van Loosdrecht, M. S. Pshenichnikov, D. Niedzialek, J. Cornil, D. Beljonne, and R. H. Friend, Science 335, 1340 (2012).

${ }^{30}$ H. van Eersel, R. A. J. Janssen, and M. Kemerink, Adv. Funct. Mater. 22, 2700 (2012).

${ }^{31}$ T. G. J. van der Hofstad, D. Di Nuzzo, M. van den Berg, R. A. J. Janssen, and S. C. J. Meskers, Adv. Energy Mater., doi: 10.1002/aenm.201200030.
${ }^{32}$ N. J. Turro, J. C. Scaiano, and V. Ramamurthy, Modern Molecular Photochemistry of Organic Molecules (University Science Books, Herndon, VA, 2010)

${ }^{33}$ T. W. Holcombe, J. E. Norton, J. Rivnay, C. H. Woo, L. Goris, C. Piliego, G. Griffini, A. Sellinger, J.-L. Bredas, A. Salleo, and J. M. J. Frechet, J. Am. Chem. Soc. 133, 12106 (2011).

${ }^{34}$ K. Vandewal, W. D. Oosterbaan, S. Bertho, V. Vrindts, A. Gadisa, L. Lutsen, D. Vanderzande, and J. V. Manca, Appl. Phys. Lett. 95, 123303 (2009).

${ }^{35}$ F. Piersimoni, S. Chambon, K. Vandewal, R. Mens, T. Boonen, A. Gadisa, M. Izquierdo, S. Filippone, B. Ruttens, J. D'Haen, N. Martin, L. Lutsen, D. Vanderzande, P. Adriaensens, and J. V. Manca, J. Phys. Chem. C 115, 10873 (2011).

${ }^{36}$ M. Lenes, G.-J. A. H. Wetzelaer, F. B. Kooistra, S. C. Veenstra, J. C. Hummelen, and P. W. M. Blom, Adv. Mater. 20, 2116 (2008).

${ }^{37}$ U.S. Patent No. 2010/0249168 A1.

${ }^{38}$ E. Wang, L. Hou, Z. Wang, S. Hellstrom, F. Zhang, O. Inganas, and M. R. Andersson, Adv. Mater. 22, 524 (2010).

${ }^{39}$ M. Bixon, J. Jortner, and J. W. Verhoeven, J. Am. Chem. Soc. 116, 7349 (1994).

${ }^{40}$ I. R. Gould, R. H. Young, L. J. Mueller, A. C. Albrecht, and S. Farid, J. Am. Chem. Soc. 116, 8188 (1994).

${ }^{41}$ Y. Yi, V. Coropceanu, and J.-L. Bredas, J. Mater. Chem. 21, 1479 (2011).

${ }^{42}$ T. Liu and A. Troisi, J. Phys. Chem. C 115, 2406 (2011).

${ }^{43}$ M. A. Loi, S. Toffanin, M. Muccini, M. Forster, U. Scherf, and M. Scharber, Adv. Funct. Mater. 17, 2111 (2007).

${ }^{44}$ M. Hallermann, I. Kriegel, E. D. Como, J. M. Berger, E. von Hauff, and J. Feldmann, Adv. Funct. Mater. 19, 3662 (2009).

${ }^{45}$ J. Kern, S. Schwab, C. Deibel, and V. Dyakonov, Phys. Status Solidi RRL 5, 364 (2011).

${ }^{46}$ B. Valuer, Molecular Fluorescence: Principles and Applications (Wiley, Weinheim, 2001).

${ }^{47}$ M. Vanecek and A. Poruba, Appl. Phys. Lett. 80, 719 (2002).

${ }^{48}$ M. Campoy-Quiles, P. G. Etchegoin, and D. D. C. Bradley, Phys. Rev. B 72, 045209 (2005).

${ }^{49}$ R. A. Street, Phys. Rev. B 84, 075208 (2011). 